\title{
Fermi Observations of the LIGO Event GW170104
}

A. Goldstein ${ }^{1}$, P. Veres ${ }^{2}$ (10, E. Burns ${ }^{3}$, L. Blackburn ${ }^{4}$ (1), M. S. Briggs ${ }^{2}$, N. Christensen ${ }^{5,6}$, W. H. Cleveland ${ }^{1}$, V. Connaughton ${ }^{1}$, T. Dal Canton ${ }^{3}$, R. Hamburg ${ }^{2}$, C. M. Hui ${ }^{7}$, P. A. Jenke ${ }^{2}$, D. Kocevski ${ }^{7,8}$, R. D. Preece ${ }^{9}$ (1), K. Siellez ${ }^{10}$, J. Veitch ${ }^{11}$, C. A. Wilson-Hodge ${ }^{7}$, N. Bhat ${ }^{2}$ (i), E. Bissaldi ${ }^{12}$ (1) M. H. Gibby ${ }^{13}$, M. M. Giles ${ }^{13}$, A. von Kienlin ${ }^{14}$ (1) B. Mailyan ${ }^{15}$, C. A. Meegan ${ }^{2}$, W. S. Paciesas ${ }^{1}$ (i), O. J. Roberts ${ }^{1}$ (i), M. Stanbro ${ }^{2}$

(Fermi-LAT Collaboration),

M. Ackermann ${ }^{16}$, M. Ajello ${ }^{17}$ (1), W. B. Atwood ${ }^{18}$, L. Baldini ${ }^{19}$ (1) , G. Barbiellini ${ }^{20,21}$, D. Bastieri ${ }^{22,23}$ (1), R. Bellazzini ${ }^{24}$ (1), R. D. Blandford ${ }^{25}$, E. D. Bloom ${ }^{25}$, R. Bonino ${ }^{26,27}$, E. Bottacini' ${ }^{25}$, T. J. Brandt ${ }^{28}$ (D) J. Bregeon $^{29}$, P. Bruel ${ }^{30}$, R. Buehler ${ }^{16}$, T. H. Burnett ${ }^{31}$, S. Buson ${ }^{28,72}$, R. A. Cameron ${ }^{25}$, P. A. Caraveo ${ }^{32}$, E. Cavazzuti ${ }^{33}$, C. Cecchi ${ }^{34,35}$, E. Charles ${ }^{25}$ (1), A. Chekhtman ${ }^{36,73}$ (1) J. Chiang ${ }^{25}$, G. Chiaro ${ }^{23}$, S. Ciprini ${ }^{34,37}$, L. R. Cominsky ${ }^{38}$, D. Costantin ${ }^{23}$, S. Cutini ${ }^{34,37}$,

F. D’Ammando ${ }^{39,40}$, , F. de Palma ${ }^{41,42}$, R. Desiante ${ }^{26,43}$ (1), S. W. Digel ${ }^{25}$, N. Di Lalla ${ }^{19}$, M. Di Mauro ${ }^{25}$ (1), L. Di Venere ${ }^{42,44}$,

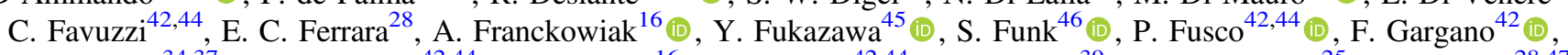

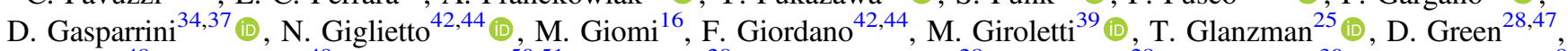

I. A. Grenier ${ }^{48}$, J. E. Grove ${ }^{49}$, L. Guillemot ${ }^{50,51}$, S. Guiriec ${ }^{28}$ (1) A. K. Harding ${ }^{28}$ (i), E. Hays ${ }^{28}$ (i), D. Horan ${ }^{30}$, G. Jóhannesson ${ }^{8,52}$,

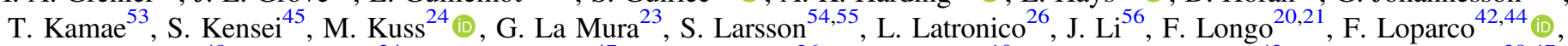

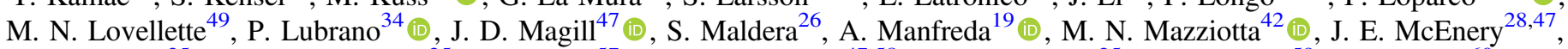
M. Meyer ${ }^{25}$ (1) , P. F. Michelson ${ }^{25}$, T. Mizuno ${ }^{57}$ (D) A. A. Moiseev ${ }^{47,58}$, M. E. Monzani2 ${ }^{25}$ (10, E. Moretti ${ }^{59}$, A. Morselli ${ }^{60}$ (i), I. V. Moskalenko ${ }^{25}$ (1) , M. Negro ${ }^{26,27}$, E. Nuss ${ }^{29}$, T. Ohsugi ${ }^{57}$, N. Omodei ${ }^{25}$ (), M. Orienti ${ }^{39}$, E. Orlando ${ }^{25}$, J. F. Ormes ${ }^{61}$, M. Palatiello ${ }^{20,21}$, V. S. Paliya ${ }^{17}$ (1) D. Paneque ${ }^{59}$, J. S. Perkins ${ }^{28}$ (i), M. Persic ${ }^{20,62}$, M. Pesce-Rollins ${ }^{24}$ (i), V. Petrosian ${ }^{25}$ (1)

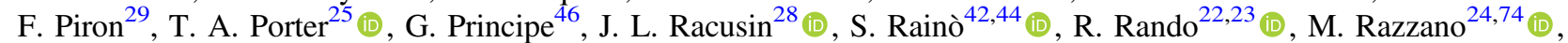
S. Razzaque ${ }^{63}$ (1) A. Reimer ${ }^{25,64}$ (D) O. Reimer ${ }^{25,64}$, T. Reposeur ${ }^{65}$, P. M. Saz Parkinson ${ }^{18,66,67}$, C. Sgrò ${ }^{24}$ (1) , E. J. Siskind ${ }^{68}$,

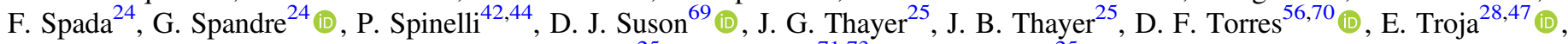
G. Vianello ${ }^{25}$ (1) K. Wood ${ }^{71,73}$, and M. Wood ${ }^{25}$

${ }^{1}$ Science and Technology Institute, Universities Space Research Association, Huntsville, AL 35805, USA

${ }^{2}$ Center for Space Plasma and Aeronomic Research, University of Alabama in Huntsville, 320 Sparkman Drive, Huntsville, AL 35899, USA ${ }^{3}$ NASA Postdoctoral Program Fellow, Goddard Space Flight Center, Greenbelt, MD 20771, USA

${ }^{4}$ Harvard-Smithsonian Center for Astrophysics, 60 Garden Street, Cambridge, MA 02138, USA ${ }^{5}$ Physics and Astronomy, Carleton College, MN, 55057, USA

${ }^{6}$ Artemis, Université Côte d'Azur, Observatoire Côte d'Azur, CNRS, CS 34229, F-06304 Nice Cedex 4, France

${ }^{7}$ Astrophysics Office, ST12, NASA/Marshall Space Flight Center, Huntsville, AL 35812, USA; kocevski@ slac.stanford.edu ${ }^{8}$ Nordita, Roslagstullsbacken 23, SE-106 91 Stockholm, Sweden

${ }^{9}$ Department of Space Science, University of Alabama in Huntsville, 320 Sparkman Drive, Huntsville, AL 35899, USA

${ }^{10}$ Center for Relativistic Astrophysics and School of Physics, Georgia Institute of Technology, Atlanta, GA 30332, USA

${ }^{11}$ University of Birmingham, Birmingham B15 2TT, UK

${ }^{12}$ Istituto Nazionale di Fisica Nucleare, Sezione di Bari, I-70126 Bari, Italy

${ }^{13}$ Jacobs Technology, Inc., Huntsville, AL, USA

${ }^{14}$ Max-Planck-Institut für extraterrestrische Physik, Giessenbachstrasse 1, D-85748 Garching, Germany

${ }^{15}$ Department of Physics and Space Sciences, Florida Institute of Technology, 150 W University Boulevard, Melbourne, FL 32901, USA ${ }^{16}$ Deutsches Elektronen Synchrotron DESY, D-15738 Zeuthen, Germany

${ }^{17}$ Department of Physics and Astronomy, Clemson University, Kinard Lab of Physics, Clemson, SC 29634-0978, USA

${ }^{18}$ Santa Cruz Institute for Particle Physics, Department of Physics and Department of Astronomy and Astrophysics, University of California at Santa Cruz, Santa Cruz, CA 95064, USA

${ }^{19}$ Università di Pisa and Istituto Nazionale di Fisica Nucleare, Sezione di Pisa, I-56127 Pisa, Italy

${ }^{20}$ Istituto Nazionale di Fisica Nucleare, Sezione di Trieste, I-34127 Trieste, Italy

${ }^{21}$ Dipartimento di Fisica, Università di Trieste, I-34127 Trieste, Italy

${ }^{22}$ Istituto Nazionale di Fisica Nucleare, Sezione di Padova, I-35131 Padova, Italy

${ }^{23}$ Dipartimento di Fisica e Astronomia "G. Galilei,”Università di Padova, I-35131 Padova, Italy

${ }^{24}$ Istituto Nazionale di Fisica Nucleare, Sezione di Pisa, I-56127 Pisa, Italy; melissa.pesce.rollins@pi.infn.it

${ }^{25}$ W. W. Hansen Experimental Physics Laboratory, Kavli Institute for Particle Astrophysics and Cosmology, Department of Physics and SLAC National Accelerator Laboratory, Stanford University, Stanford, CA 94305, USA; nicola.omodei@ stanford.edu, giacomov@slac.stanford.edu

${ }_{26}^{26}$ Istituto Nazionale di Fisica Nucleare, Sezione di Torino, I-10125 Torino, Italy

${ }^{27}$ Dipartimento di Fisica, Università degli Studi di Torino, I-10125 Torino, Italy ${ }^{28}$ NASA Goddard Space Flight Center, Greenbelt, MD 20771, USA

${ }^{29}$ Laboratoire Univers et Particules de Montpellier, Université Montpellier, CNRS/IN2P3, F-34095 Montpellier, France

${ }^{30}$ Laboratoire Leprince-Ringuet, École polytechnique, CNRS/IN2P3, F-91128 Palaiseau, France

${ }^{31}$ Department of Physics, University of Washington, Seattle, WA 98195-1560, USA

${ }^{32}$ INAF-Istituto di Astrofisica Spaziale e Fisica Cosmica Milano, via E. Bassini 15, I-20133 Milano, Italy ${ }^{3}$ Italian Space Agency, Via del Politecnico snc, I-00133 Roma, Italy

${ }_{35}^{34}$ Istituto Nazionale di Fisica Nucleare, Sezione di Perugia, I-06123 Perugia, Italy

35 Dipartimento di Fisica, Università degli Studi di Perugia, I-06123 Perugia, Italy

${ }^{36}$ College of Science, George Mason University, Fairfax, VA 22030, USA

${ }^{37}$ ASI Space Science Data Center, Via del Politecnico snc, 00133 Roma, Italy

${ }^{38}$ Department of Physics and Astronomy, Sonoma State University, Rohnert Park, CA 94928-3609, USA ${ }^{39}$ INAF Istituto di Radioastronomia, I-40129 Bologna, Italy

${ }^{40}$ Dipartimento di Astronomia, Università di Bologna, I-40127 Bologna, Italy 


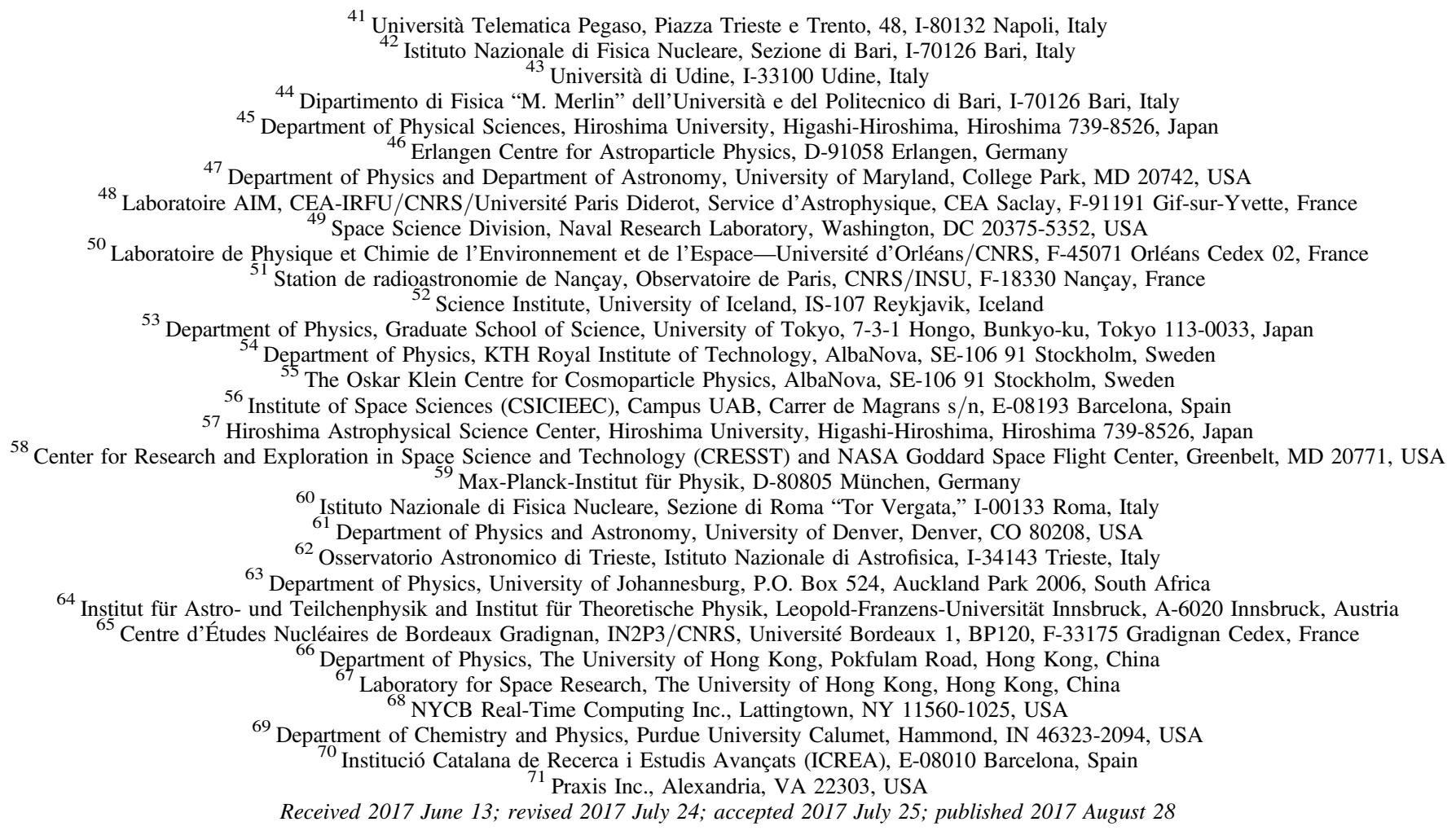

\begin{abstract}
We present the Fermi Gamma-ray Burst Monitor (GBM) and Large Area Telescope (LAT) observations of the LIGO binary black hole merger (BBH) event GW170104. No candidate electromagnetic counterpart was detected by either GBM or LAT. A detailed analysis of the GBM and LAT data over timescales from seconds to days covering the Laser Interferometer Gravitational-wave Observatory (LIGO) localization region is presented. The resulting flux upper bound from the GBM is $(5.2-9.4) \times 10^{-7} \mathrm{erg} \mathrm{cm}^{-2} \mathrm{~s}^{-1}$ in the $10-1000 \mathrm{keV}$ range and from the LAT is $(0.2-90) \times 10^{-9} \mathrm{erg} \mathrm{cm}^{-2} \mathrm{~s}^{-1}$ in the $0.1-1 \mathrm{GeV}$ range. We also describe the improvements to our automated pipelines and analysis techniques for searching for and characterizing the potential electromagnetic counterparts for future gravitational-wave events from Advanced LIGO/Virgo.
\end{abstract}

Key words: gamma rays: general - gravitational waves - methods: observational

\section{Introduction}

The detection of gravitational waves (GWs) has opened a new window to the cosmos and paved the way to a new era of multi-messenger astronomy. The first detected signals by Advanced Laser Interferometer Gravitational-wave Observatory (LIGO), GW150914 and GW151226 (Abbott et al. 2016a, 2016b), are compatible with the coalescence of highmass binary black holes (BBHs). The detection of a third BBH merger, GW170104 (Abbott et al. 2017; $t_{\mathrm{GW}}=2017-01-04$ 10:11:59 UTC), with component masses of $\sim 31 M_{\odot}$ and $\sim 19 M_{\odot}$, adds to the growing sample of BBH merger events. The component masses and the post-merger mass $\left(\sim 49 M_{\odot}\right)$ of GW170104 falls between those of GW151226 and GW150914, while the estimated distance of $\sim 880 \mathrm{Mpc}(z \approx 0.18)$ is roughly twice as far compared to the previous detections. GW binary coalescence events are also predicted to result from the mergers of other compact object binary systems such as

\footnotetext{
72 NASA Postdoctoral Program Fellow.

73 Resident at Naval Research Laboratory, Washington, DC 20375, USA.

74 Funded by contract FIRB-2012-RBFR12PM1F from the Italian Ministry of Education, University and Research (MIUR).
}

neutron star-black hole (NS-BH) and neutron star-neutron star (NS-NS). Short gamma-ray bursts (sGRBs) are thought to be associated with such systems (Eichler et al. 1989; Narayan et al. 1992; Lee \& Ramirez-Ruiz 2007), which strongly motivate searches for electromagnetic (EM) counterparts to $\mathrm{GW}$ events.

The Fermi Gamma-ray Burst Monitor (GBM; Meegan et al. 2009 ) is the most prolific detector of sGRBs (Bhat et al. 2016; 40 per year); however, it localizes these sources with uncertainties of the order of a few degrees, making the follow-up by some instruments at other wavelengths challenging. The Fermi Large Area Telescope (LAT; Atwood et al. 2009) has a lower detection rate for sGRBs but can provide $0.2^{\circ}-0.3^{\circ}$ localizations. In the case of a detection of an EM counterpart, the LAT could substantially reduce the localization uncertainty, facilitating follow-up at other wavelengths. The two instruments on board the Fermi satellite are complementary and uniquely capable of providing all-sky observations from hard X-rays to high-energy gamma-rays in normal survey operations. Together, they cover the entire localization probability maps of gravitational-wave events (Ackermann et al. 2016; Racusin et al. 2017) within hours of their detections. 


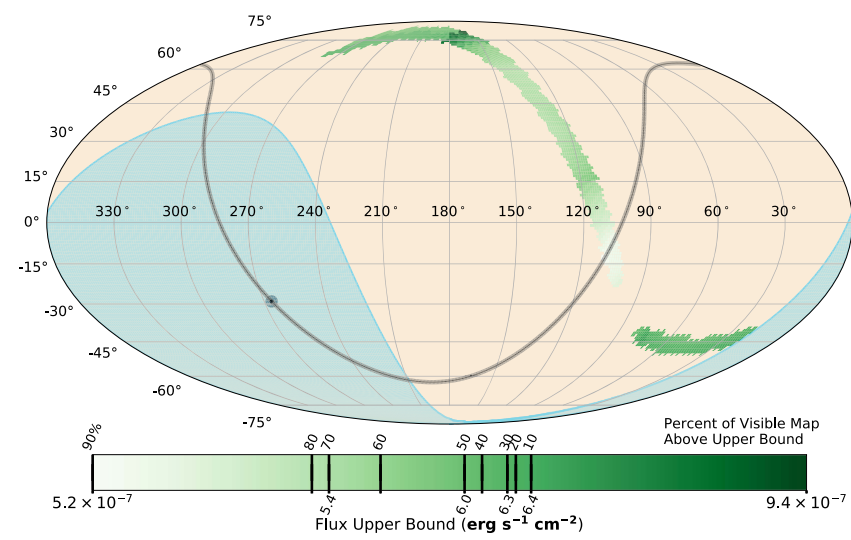

Figure 1. The $1 \mathrm{~s}$ flux upper bound by GBM covering the $90 \%$ credible region of the LIGO sky map. The Earth occultation region is shown in blue, and the Galactic plane is shown as the gray line with the Galactic center demarcated with a gray circle. The marks on the color bar indicate the percentage of the visible LIGO sky map that is above a given upper bound (e.g., $70 \%$ of the visible map has an upper bound $\left.\geqslant 5.4 \times 10^{-7} \mathrm{erg} \mathrm{cm}^{-2} \mathrm{~s}^{-1}\right)$.

\section{Observations and Data Analysis}

\section{1. $G B M$}

The Fermi GBM has 12 sodium iodide (Na I) detectors and 2 bismuth germanate (BGO) detectors sensitive over an energy range of $8 \mathrm{keV}-40 \mathrm{MeV}$ with 128-channel spectral resolution (Meegan et al. 2009). The detectors observe the full unocculted sky $(\sim 70 \%)$ and operate continuously except during passage of Fermi through the South Atlantic Anomaly (SAA), so that the average duty cycle for observing a given sky location is $\sim 60 \%$. Since late 2012 , GBM has provided continuous time-tagged event data (CTTE) with a resolution down to $2 \mu \mathrm{s}$, which, together with the large spectral range and sky coverage, makes GBM the most prolific detector currently in operation for prompt GRB observations.

\subsubsection{GBM Signal Searches}

An automatic, ground-based search of the GBM CTTE data for sGRBs is currently running promptly after the data are downlinked. This search, which we refer to here as the untargeted search, is similar to the flight software algorithms that GBM uses for triggering on GRBs, which look for simultaneous excesses in two GBM Na I detectors. This search rebins the CTTE data into time series with 18 bin widths from $64 \mathrm{~ms}$ to $30.72 \mathrm{~s}$ and four phase offsets for each bin width. These time series are created for four different energy ranges that were optimized for finding weak triggered sGRBs: nominally $30-500 \mathrm{keV}, \quad 50-500 \mathrm{keV}$, $100-500 \mathrm{keV}$, and $100 \mathrm{keV}-1 \mathrm{MeV}$. The background estimation is performed via cubic spline fitting, and intervals of data are removed from consideration if the background fit deviates significantly from the observed background. While the in-orbit triggering algorithms require signals to reach $4.5 \sigma$ in two separate detectors, the untargeted search flags any signals that exceed $2.5 \sigma$ in one detector and $1.25 \sigma$ in another. A pretrial $p$-value is calculated for the flagged candidate and compared against a predetermined $p$-value threshold to determine whether a candidate signal has been found. Archival CTTE data have been searched starting from 2013 January, finding $\approx 80$ candidate sGRBs per year, in addition to the 40 sGRBs found by in-orbit triggering. ${ }^{75}$

\footnotetext{
75 https://gammaray.nsstc.nasa.gov/gbm/science/sgrb_search.html
}

The GBM targeted search pipeline (Blackburn et al. 2015), which operates using a seed time and an optional seed sky map, was updated in preparation for the second observing run of Advanced LIGO (O2) to produce an overall lower false-alarm rate (FAR) and to be more sensitive to sGRB-like signals (Goldstein et al. 2016). The improvements implemented include replacing the "hard" spectral template with a more physically and observationally representative cutoff power-law spectrum, increasing the data temporal resolution, improving the background estimation, and utilizing localization information in the ranking statistic.

\subsubsection{GBM Results}

The GBM was observing $~ 82 \%$ of the LIGO sky map at the time of the LIGO trigger, and no onboard GBM trigger was recorded close in time to GW170104. The nearest triggers were more than $13 \mathrm{hr}$ preceding and more than $10 \mathrm{hr}$ following GW170104, and both of these triggers were due to steep nonastrophysical rate increases near the boundary of the SAA just before the detectors were turned off for passage. The untargeted search did not find any untriggered sGRB candidate on January 4. The nearest candidate preceding GW170104 was more than $15 \mathrm{hr}$ earlier, and the nearest candidate following GW170104 was about 8 days later.

The targeted search was run over a 1 minute window centered at $t_{\mathrm{GW}}$, searching for any signal on timescales from $0.256 \mathrm{~s}$, increasing by factors of two up to $8.192 \mathrm{~s}$ (six timescales). As reported in Burns et al. (2017), no credible significant candidate was found. The most significant result from the search was on the longest timescale with the "soft" spectral template, which the search found to be marginally consistent with part of the LIGO sky map. Further inspection of the count-rate light curves for each detector reveal a slow-rising bump that begins $\sim 100 \mathrm{~s}$ before $t_{\mathrm{GW}}$ and has a duration of $\sim 300 \mathrm{~s}$. This bump, which is strongest in the GBM Na I channel $1(12-25 \mathrm{keV})$ and does not appear above channel $2(50 \mathrm{keV})$, is consistent with a low-level increase in magnetospheric activity as Fermi proceeds through its orbit.

Using the GBM, we can set an upper bound on the impulsive flux expected from an sGRB-like signal. The current technique is to estimate the background in the same way that the targeted search does (unbinned Poisson maximum likelihood over a sliding window) and calculate the $3 \sigma$ upper bound in the count rate as three times the standard deviation of the background count rate. The count-rate upper bound can then be converted to a flux upper bound by assuming a photon model, folding it through the detector response and fitting the amplitude to determine the associated flux upper bound. We note that this technique produces a conservative upper limit because it utilizes a single GBM detector rather than all detectors over the entire LIGO map, which would be significantly more computationally challenging. We evaluate the limits in this way over the $90 \%$ credible region of the visible LIGO sky map by splitting up the map into a set of equally spaced regions on the sky and considering the GBM Na I detector with the smallest pointing angle to the center of each region. Performing this technique over the targeted search window, we estimate the $1 \mathrm{~s}$ averaged flux upper bound assuming a cutoff power law with $E_{\text {peak }}=566 \mathrm{keV}$ and photon index of -0.42 , which represents the peak density of the spectral parameter distribution for GBM-triggered sGRBs. $^{76}$ The resulting spatially

\footnotetext{
76 https://heasarc.gsfc.nasa.gov/W3Browse/fermi/fermigbrst.html
} 
dependent upper bound is shown in Figure 1 and ranges from $(5.2-9.4) \times 10^{-7} \mathrm{erg} \mathrm{s}^{-1} \mathrm{~cm}^{-2}$ in the $10-1000 \mathrm{keV}$ energy band for the visible LIGO sky map. Consider that only the $50 \%$ credible region of the sky map removes the least-stringent region of the GBM upper bound, resulting in an upper bound range of (5.2-7.6) $\times 10^{-7} \mathrm{erg} \mathrm{s}^{-1} \mathrm{~cm}^{-2}$. These upper bounds are below $90 \%-99 \%$ of GBM-triggered sGRBs with a similar spectrum to the model used for the upper bound estimation. Based on the distance of GW170104, $D_{L} \approx 880 \mathrm{Mpc}$, $z \approx 0.18$, the flux upper bound translates to a K-corrected luminosity upper bound range of (5.5-9.9) $\times 10^{49} \mathrm{erg} \mathrm{s}^{-1}$ in the approximately bolometric $1-10^{4} \mathrm{keV}$ energy range typically reported for GRBs.

In addition to upper bounds for impulsive emission, we searched for any long-lasting or persistent emission related to GW170104 using the Earth occultation technique (EOT; Wilson-Hodge et al. 2012). The search was performed for a two-day interval centered on the $t_{\mathrm{GW}}$ time for any sources within the $90 \%$ LIGO localization credible region. No new sources were detected during this interval and no significant change in flux was recorded for any of the sources that GBM typically observes with the EOT. A search was also performed for a 30 day period after the trigger, and again no new source was detected.

\section{2. $L A T$}

The LAT (Atwood et al. 2009) is a pair conversion telescope comprising a $4 \times 4$ array of silicon strip trackers and tungsten converters together with cesium iodide (CsI) calorimeters covered by a segmented anticoincidence detector to reject charged-particle background events. The LAT is sensitive in the energy range from $20 \mathrm{MeV}$ to more than $300 \mathrm{GeV}$ with a field of view (FoV) of $2.4 \mathrm{sr}$, observing the entire sky every two orbits $(3 \mathrm{hr})$ by rocking north and south about the orbital plane on alternate orbits (Atwood et al. 2009). The LAT detects about 15 GRBs per year above $100 \mathrm{MeV}, \sim 2-3$ of which are sGRBs with localization precisions on the order of $\sim 10$ arcmin (Vianello et al. 2015). This latter class of GRBs, when detected above $100 \mathrm{MeV}$, has a substantially longer duration with respect to their $\mathrm{keV}-\mathrm{MeV}$ emission and is thought to be related to the afterglow phase. The LAT is the only instrument that has detected and localized an sGRB during its afterglow phase starting from the GBM localization (Ackermann et al. 2010). Furthermore, the LAT can substantially reduce the localization uncertainties with respect to GBM, aiding follow-up at other wavelengths.

\subsubsection{The LAT Signal Searches}

Several automatic programs search for transient events over a wide range of timescales in the LAT data. The Fermi automatic science processing (ASP) pipeline and the Fermi All-sky Variability Analysis (FAVA) ${ }^{77}$ are used to search for excess emission on hour-to-week-long timescales. The ASP pipeline performs a blind search for sources on all-sky count maps constructed from the event data acquired on 6 and $24 \mathrm{hr}$ timescales. Candidate flaring sources are then fit using a standard likelihood analysis with the model including nearby known sources and the Galactic and isotropic diffuse contributions.

\footnotetext{
77 https://fermi.gsfc.nasa.gov/ssc/data/access/lat/FAVA/
}

These candidate sources are then characterized and matched to known sources, allowing for the detection of flaring cataloged sources as well as new unassociated sources. FAVA is a blind photometric analysis in which a grid of regions covering the entire sky is searched over $24 \mathrm{hr}$ and 1 week timescales for deviations from the expected flux based on the observed longterm mission-averaged emission (Ackermann et al. 2013). This allows the FAVA search to be independent of any model of the gamma-ray sky, and therefore complementary to the standard likelihood analyses.

As described in Ackermann et al. (2016), Racusin et al. (2017), and, more specifically, in Vianello et al. (2017), we have designed specific methods for the follow-up searches in the LAT data of EM counterparts to GW events. The fixed and adaptive time windows are two custom search methods that we run on the LIGO localization maps. In the first case, we search for high-energy gamma-ray emission on a set of fixed time windows $(\approx 10 \mathrm{ks}$ long) around the GW trigger. For each time window, we select all the pixels ${ }^{78}$ that were observable by the LAT within the $90 \%$ containment of the LIGO localization map and perform a likelihood analysis in an $8^{\circ}$ radius region of interest (ROI). In the latter, we optimize the time window for the analysis based on when the pixel becomes observable by the LAT. For each pixel we further select only the interval that contains the GW trigger time, or the one immediately after (if the center of the pixel was outside of the LAT FoV at the GW trigger time). Once we have defined these adaptive time windows, we perform a likelihood analysis for each pixel. This analysis is therefore optimized for the assumption that the source emitted gammarays at the time of the $\mathrm{GW}$ event. In both cases, we use the Pass 8 P8_TRANSIENTO10E_V6 events class and the corresponding instrument response functions. We perform the standard unbinned maximum likelihood analysis used by the LAT Collaboration from 0.1 to $1 \mathrm{GeV}$. Our likelihood model includes all sources (point-like and extended) from the 3FGL catalog of LAT sources (Acero et al. 2015), as well as the Galactic and isotropic diffuse templates provided in Acero et al. (2016). To model the new possible source we assume a power-law spectrum with a photon index of -2 , which is typical for GRB afterglows at LAT energies. We employ a likelihood-ratio test (Neyman \& Pearson 1928) to quantify whether the existence of a new source is statistically warranted. In doing so, we form a test statistic (TS) described in Vianello et al. (2017) to reject a null hypothesis of no signal associated with the GW trigger. As is standard for LAT analysis, we choose to reject the null hypothesis when the TS is greater than 25, roughly equivalent to a $5 \sigma$ rejection criterion for a single search.

In preparation for the LIGO O2 science runs, we have implemented several improvements to our GW follow-up searches. These include an analysis pipeline to automatically process LAT data every time a LIGO/Virgo gamma-ray coordinates network $(\mathrm{GCN})$ is received, running both the fixed time window search and the adaptive time window search methods.

\subsubsection{LAT Observations of GW170104}

The LAT was favorably oriented toward GW170104 at the time of the trigger, $t_{\mathrm{GW}}$, covering $55 \%$ of the LIGO localization map at $t_{\mathrm{GW}}$, and within $5 \mathrm{ks}$ from $t_{\mathrm{GW}}$ the LAT

\footnotetext{
${ }^{78}$ In our analysis, the average separation between pixels is $\approx 0.5^{\circ}$.
} 
had observed $100 \%$ of the LIGO probability map. The LAT then continued to observe the entire LIGO localization region throughout normal sky-survey operations in the following days. We performed a fixed time window search from $t_{\mathrm{GW}}$ to $t_{\mathrm{GW}}+10 \mathrm{ks}$ and found no credible candidate counterparts. We thus performed the upper bound computation described in Vianello et al. (2017) to evaluate the 95\% upper bound in the $0.1-1 \mathrm{GeV}$ range for the fixed time interval and found a value of $\mathrm{F}_{u l, 95}=3 \times 10^{-10} \mathrm{erg} \mathrm{cm}^{-2} \mathrm{~s}^{-1}$.

The adaptive time window analysis also yielded no significant excesses, and no new sources were detected above a $5 \sigma$ detection threshold. The flux upper bounds in the 0.1-1 GeV range for the portion of the LIGO localization contour containing $90 \%$ of the probability during the adaptive time window are shown in Figure 2. The values for the flux upper bounds from this analysis range from $0.2-90 \times 10^{-9} \mathrm{erg}$ $\mathrm{cm}^{-2} \mathrm{~s}^{-1}$.

Given that we do not know the nature of the potential EM counterpart to a BBH merger, we also examined the ASP products during the 6 and $24 \mathrm{hr}$ intervals containing $t_{\mathrm{GW}}$. We also performed a dedicated FAVA search over a $24 \mathrm{hr}$ and 1 week intervals centered on $t_{\mathrm{GW}}$. The two searches found two flaring sources with significance above $5 \sigma$ within the LIGO localization map. Follow-up likelihood analyses of both sources found that their positions were consistent with flatspectrum radio quasars (S5 1044+71 and OK 630), which have shown previous evidence of flaring activity. No new unassociated flaring sources were detected within the LIGO localization contour.

\section{Summary}

It is unclear how BBH mergers can be followed by BHaccretion disk systems that are conventionally invoked (e.g., Berger 2014) to channel the gravitational energy into EM radiation. For GW150914, Connaughton et al. (2016) reported a possible counterpart, which increased interest in scenarios by which BBH mergers may yield significant gamma-ray emission. However, all current models suffer from nonnegligible inconsistencies (e.g., Lyutikov 2016), and for this reason even upper bounds like the ones presented here contribute important information to the puzzle. Some models (Loeb 2016; Woosley 2016) involve the presence of a common envelope or a circumbinary disk and have been also used to explain possible neutrino emission (Moharana et al. 2016; Janiuk et al. 2017). According to Perna et al. (2016), an sGRB might result from the BBH merger triggering the accretion of a disk around one of the BHs in the final seconds before the coalescence, but Kimura et al. (2017) find it is difficult to have the disk survive down to seconds before the coalescence as required. An efficient way to power EM emission is the Blandford-Znajek (Blandford \& Znajek 1977) process of electromagnetic extraction of mechanical energy ( $\mathrm{Li}$ et al. 2016; Veres et al. 2016). Lyutikov (2016), however, pointed out that the EM luminosity associated with GW150914 would require magnetic fields of $\sim 10^{12} \mathrm{G}$, astrophysically implausible for an environment around a $\mathrm{BH}$.

Based on the general arguments listed in Racusin et al. (2017), non-detection of an EM counterpart for GW170104 does not rule out the association between GW150914 and GW150914-GBM. These arguments are as follows. (a) The source may have been occulted by the Earth from Fermi at $t_{\mathrm{GW}}$ ( $\sim 18 \%$ of the LIGO map was occulted; Burns et al. 2017).
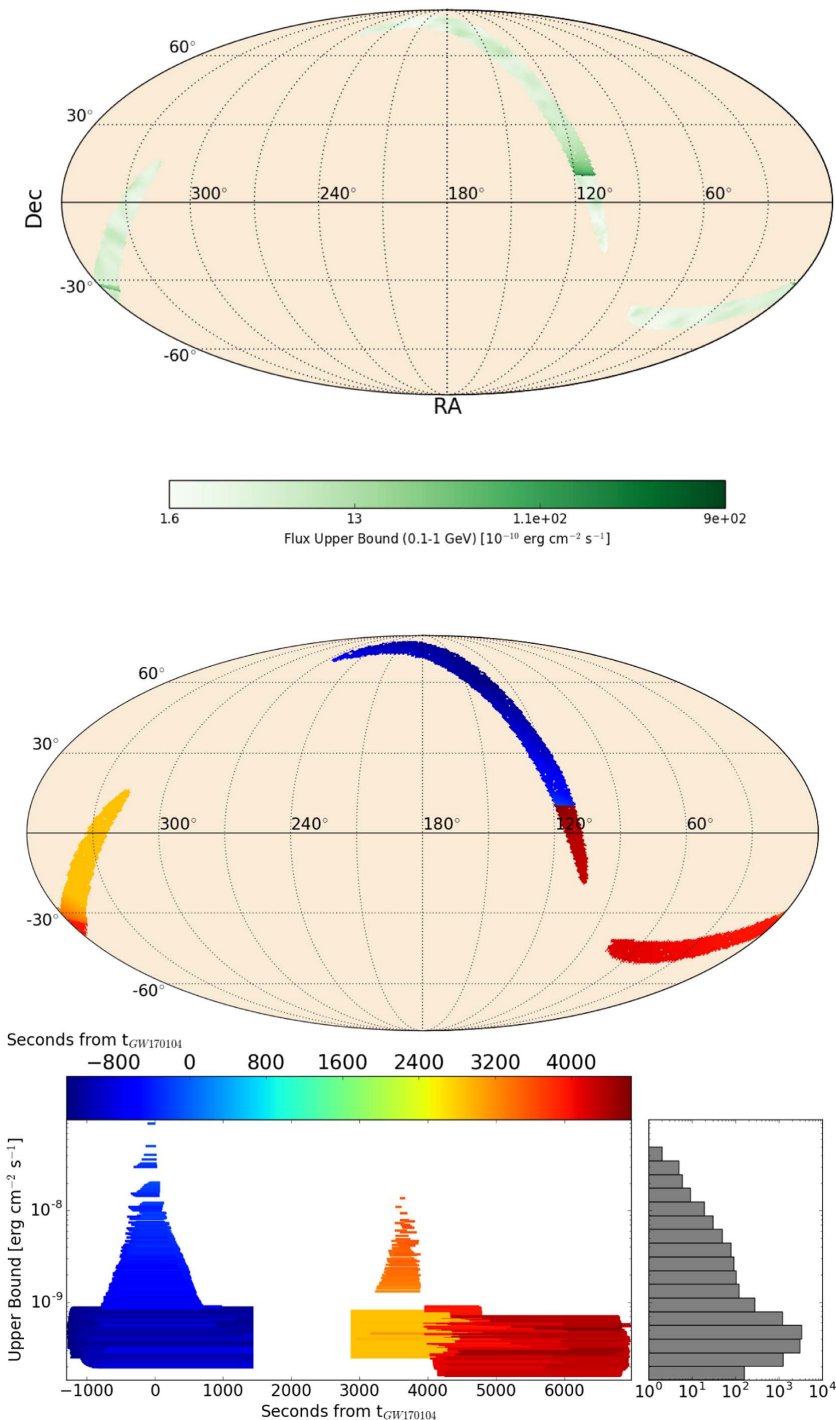

Figure 2. Flux upper bound map resulting from the time adaptive analysis (top), the entry time into the LAT FoV relative to the $t_{\mathrm{GW}}$ for each pixel within the LIGO localization contour (middle), and the upper bound light curves for each ROI (bottom). The horizontal bars in the bottom panel correspond to a pixel within $90 \%$ of the LIGO localization map and the $y$-scale represents the values of the LAT upper bounds. The position of these bars along the time-axis coincides with the interval of time used in the analysis. The color of each bar indicates the time when the $8^{\circ} \mathrm{ROI}$ entered the LAT FoV and matches the color of the pixel in the middle panel. The horizontal histogram displays the distribution of upper bounds. The gap between $\sim 1500$ and $\sim 3000 \mathrm{~s}$ is due to the passage of the satellite in the South Atlantic Anomaly.

(b) The GBM background rates are higher for GW170104 (average of 113 counts $\mathrm{s}^{-1}$ per detector, or $11 \%$, higher compared to GW150914-GBM). Note that the estimated flux for the $\sim 1 \mathrm{~s}$ long signal GW150914-GBM $\left(\sim 2 \times 10^{-7} \mathrm{erg} \mathrm{s}^{-1}\right.$ $\mathrm{cm}^{-2}$; Connaughton et al. 2016) is below the GBM flux upper limit we estimate here, indicating that it is unlikely that GW150914-GBM could be detected with this increased background rate. (c) A jet, if present, was pointing away from us. (d) The EM emission associated with GW170104 was dimmer than for GW150914 (e.g., related to the lower BH masses and the larger source distance).

GW170104 is the third BBH merger detected by Advanced LIGO. While no conventional model predicts EM counterparts 
for these sources, our observations are important in two respects. (a) We improve and test the search algorithms in anticipation of $\mathrm{GW}$ events where we do expect an EM counterpart (e.g., a binary merger with at least one NS component). (b) We provide flux upper bound constraints for the unexpected scenario where BBH mergers are accompanied by gamma-rays.

The Fermi-GBM collaboration acknowledges support for GBM development, operations, and data analysis from NASA in the United States and BMWi/DLR in Germany. The FermiLAT Collaboration acknowledges support for LAT development, operation, and data analysis from NASA and DOE (United States), CEA/Irfu and IN2P3/CNRS (France), ASI and INFN (Italy), MEXT, KEK, and JAXA (Japan), and the K. A. Wallenberg Foundation, the Swedish Research Council and the National Space Board (Sweden). Science analysis support in the operations phase from INAF (Italy) and CNES (France) is also gratefully acknowledged. This work performed in part under DOE Contract DE-AC02-76SF00515.

\section{ORCID iDs}

P. Veres (1) https://orcid.org/0000-0002-2149-9846

L. Blackburn (1) https://orcid.org/0000-0002-9030-642X

R. D. Preece (1) https://orcid.org/0000-0003-1626-7335

N. Bhat (i) https://orcid.org/0000-0001-7916-2923

E. Bissaldi (10) https://orcid.org/0000-0001-9935-8106

A. von Kienlin (10 https://orcid.org/0000-0002-0221-5916

W. S. Paciesas 니 https://orcid.org/0000-0002-2481-5947

O. J. Roberts (10 https://orcid.org/0000-0002-7150-9061

M. Ajello (다 https://orcid.org/0000-0002-6584-1703

L. Baldini (1) https://orcid.org/0000-0002-9785-7726

D. Bastieri (i) https://orcid.org/0000-0002-6954-8862

R. Bellazzini iㅏ https://orcid.org/0000-0002-2469-7063

T. J. Brandt (10) https://orcid.org/0000-0003-4087-1786

E. Charles (1) https://orcid.org/0000-0002-3925-7802

A. Chekhtman (1) https://orcid.org/0000-0002-6643-9556

F. D'Ammando (1) https://orcid.org/0000-0001-7618-7527

R. Desiante (1) https://orcid.org/0000-0002-5558-6956

M. Di Mauro (1) https://orcid.org/0000-0001-7801-7484

A. Franckowiak (1) https://orcid.org/0000-0002-5605-2219

Y. Fukazawa (1) https://orcid.org/0000-0002-0921-8837

S. Funk (1) https://orcid.org/0000-0002-2012-0080

P. Fusco (1) https://orcid.org/0000-0002-9383-2425

F. Gargano (i) https://orcid.org/0000-0002-5055-6395

D. Gasparrini (1) https://orcid.org/0000-0002-5064-9495

N. Giglietto (1) https://orcid.org/0000-0002-9021-2888

M. Giroletti (1) https://orcid.org/0000-0002-8657-8852

T. Glanzman (1) https://orcid.org/0000-0001-9649-3871

S. Guiriec (i) https://orcid.org/0000-0001-5780-8770

A. K. Harding (1) https://orcid.org/0000-0001-6119-859X

E. Hays (i) https://orcid.org/0000-0002-8172-593X

M. Kuss (1) https://orcid.org/0000-0003-1212-9998

F. Loparco (1) https://orcid.org/0000-0002-1173-5673

P. Lubrano (1) https://orcid.org/0000-0003-0221-4806

J. D. Magill (1) https://orcid.org/0000-0001-9231-2965

A. Manfreda (1) https://orcid.org/0000-0002-0998-4953
M. N. Mazziotta (1) https://orcid.org/0000-0001-9325-4672

M. Meyer (1) https://orcid.org/0000-0002-0738-7581

T. Mizuno (1) https://orcid.org/0000-0001-7263-0296

M. E. Monzani (1) https://orcid.org/0000-0002-8254-5308

A. Morselli (1) https://orcid.org/0000-0002-7704-9553

I. V. Moskalenko (i) https://orcid.org/0000-0001-6141-458X

N. Omodei (1) https://orcid.org/0000-0002-5448-7577

V. S. Paliya (1) https://orcid.org/0000-0001-7774-5308

J. S. Perkins (10) https://orcid.org/0000-0001-9608-4023

M. Pesce-Rollins (1) https://orcid.org/0000-0003-1790-8018

V. Petrosian (10) https://orcid.org/0000-0002-2670-8942

T. A. Porter (1) https://orcid.org/0000-0002-2621-4440

J. L. Racusin (1) https://orcid.org/0000-0002-4744-9898

S. Rainò (i) https://orcid.org/0000-0002-9181-0345

R. Rando (1) https://orcid.org/0000-0001-6992-818X

M. Razzano (1) https://orcid.org/0000-0003-4825-1629

S. Razzaque (다 https://orcid.org/0000-0002-0130-2460

A. Reimer (1) https://orcid.org/0000-0001-6953-1385

C. Sgrò (i) https://orcid.org/0000-0001-5676-6214

G. Spandre (i) https://orcid.org/0000-0003-0802-3453

D. J. Suson (1) https://orcid.org/0000-0003-2911-2025

D. F. Torres (1) https://orcid.org/0000-0002-1522-9065

E. Troja (i) https://orcid.org/0000-0002-1869-7817

G. Vianello (1) https://orcid.org/0000-0002-2553-0839

\section{References}

Abbott, B. P., Abbott, R., Abbott, T. D., et al. 2016a, PRL, 116, 061102 Abbott, B. P., Abbott, R., Abbott, T. D., et al. 2016b, PRL, 116, 241103 Abbott, B. P., Abbott, R., Abbott, T. D., et al. 2017, PRL, 118, 221101 Acero, F., Ackermann, M., Ajello, M., et al. 2015, ApJS, 218, 23 Acero, F., Ackermann, M., Ajello, M., et al. 2016, ApJS, 223, 26 Ackermann, M., Ajello, M., Albert, A., et al. 2013, ApJ, 771, 57 Ackermann, M., Ajello, M., Albert, A., et al. 2016, ApJ, 823, 2

Ackermann, M., Asano, K., Atwood, W. B., et al. 2010, ApJ, 716, 1178 Atwood, W. B., Abdo, A. A., Ackermann, M., et al. 2009, ApJ, 697, 1071 Berger, E. 2014, ARA\&A, 52, 43

Bhat, N. P., Meegan, C. A., von Kienlin, A., et al. 2016, ApJS, 223, 28

Blackburn, L., Briggs, M. S., Camp, J., et al. 2015, ApJS, 217, 8

Blandford, R. D., \& Znajek, R. L. 1977, MNRAS, 179, 433

Burns, E., Blackburn, L., Briggs, M. S., et al. 2017, GCN, 20365, https://gcn. gsfc.nasa.gov/other/G268556.gcn3

Connaughton, V., Burns, E., Goldstein, A., et al. 2016, ApJL, 826, L6

Eichler, D., Livio, M., Piran, T., \& Schramm, D. N. 1989, Natur, 340, 126 Goldstein, A., Burns, E., Hamburg, R., et al. 2016, arXiv:1612.02395

Janiuk, A., Bejger, M., Charzyński, S., \& Sukova, P. 2017, NewA, 51, 7

Kimura, S. S., Takahashi, S. Z., \& Toma, K. 2017, MNRAS, 465, 4406

Lee, W. H., \& Ramirez-Ruiz, E. 2007, NJPh, 9, 17

Li, X., Hu, Y.-M., Fan, Y.-Z., \& Wei, D.-M. 2016, ApJ, 827, 75

Loeb, A. 2016, ApJL, 819, L21

Lyutikov, M. 2016, arXiv:1602.07352

Meegan, C., Lichti, G., Bhat, P. N., et al. 2009, ApJ, 702, 791

Moharana, R., Razzaque, S., Gupta, N., \& Mészáros, P. 2016, PhRvD, 93, 123011

Narayan, R., Paczynski, B., \& Piran, T. 1992, ApJL, 395, L83

Neyman, J., \& Pearson, E. S. 1928, Biometrika, 20, 175

Perna, R., Lazzati, D., \& Giacomazzo, B. 2016, ApJL, 821, L18

Racusin, J. L., Burns, E., Goldstein, A., et al. 2017, ApJ, 835, 1

Veres, P., Preece, R. D., Goldstein, A., et al. 2016, ApJL, 827, L34

Vianello, G., Omodei, N. \& Fermi/LAT collaboration 2015, arXiv:1502.03122

Vianello, G., Omodei, N., Chiang, J., \& Digel, S. 2017, ApJL, 841, L16

Wilson-Hodge, C. A., Case, G. L., Cherry., M. L., et al. 2012, ApJS, 201, 33

Woosley, S. E. 2016, ApJL, 824, L10 